\title{
Applicability of Non-Rigid Medical Image Registration using Moving Least Squares
}

\author{
Ms.Hema P Menon \\ Department of Computer Science and Engineering \\ Amrita School of Engineering, Coimbatore-641105
}

\author{
Dr.K.A.Narayanankutty \\ Department of Electronics and Comm. Engineering \\ Amrita School of Engineering, Coimbatore-641105
}

\begin{abstract}
A drawback of the non-rigid registration is its unpredictable nature of the deformation on the target image. Mapping every point on images can cause deformations even to regions, which are expected to remain rigid. A non-rigid registration is therefore desirable, that produces only local deformations where needed, while still preserving the overall rigidity. This work focuses on one such method called the Moving Least Squares (MLS) transformation and compares the results with Thin Plate Splines (TPS). An intensity based non-rigid registration algorithm is applied apriory, if the input medical images are from two different patients in order to facilitate for the selection of homologous control points in them. We compare the performance of both the techniques by calculating the Target Registration Error (TRE) at certain points and results are encouraging.
\end{abstract}

\section{Keywords}

Moving Least Squares, Non-rigid medical image registration, Thin Plate Splines, As-rigid-as-possible transformations.

\section{INTRODUCTION}

Image registration, a processing requirement for combining images (i.e., image fusion), is used extensively in the medical field for monitoring disease progression, image guided surgery, studying brain shift after surgery and developing medical atlases. The process for image registration involves two images namely, the source and the target images, and finding the best deformation field to align the two images. Various features like points, line segments and intensities can be used as the basis for the registration. Functions used to map the source image to the target image are called transformation functions. It is classified as rigid or non-rigid, based on the transformation function used. Unlike rigid registrations, where the distances between all points remain constant before and after the registration, a non-rigid registration involve more complex computations like local stretching and scaling to map the two images which might generate unpredictable deformations. It is not possible to exactly specify the mapping of each point in the source image to the target image. It would thus be good to have a non-rigid registration technique that produces local deformations

only

where needed while still preserving the overall rigidity of the transformation as much as possible called as-rigid-aspossible methods. Fortunately such techniques exist, and this work focuses on one such method called the MLS transformation [2]. This technique is relatively recent and is better known for surface reconstruction and in computer graphics for image deformation and morphing. Here, we are exploring the applicability of the MLS technique as a point based non-rigid registration technique. Methods of MLS using affine, similarity transformations and rigid transformations are presented elsewhere [22]. The results are compared with TPS method, which is another widely used technique both qualitatively and quantitatively.

The source and target images considered for registration are of the same modality. To evaluate the performance of the MLS technique, 2D MR brain images from the same patient and different patients were considered as the input to the system. If the source and target images are from the same patient, then homologous control points are selected from the respective images and are passed to each of the registration algorithm to get the transformed source. If the input images are from two different patients, an intensity based non-rigid registration algorithm is used for the selection of homologous control points. Then the selected control points are applied to each of the point based technique to obtain the registered source. During this process we can compare the performance of both the techniques by calculating the TRE at certain points in the image other than the control points.

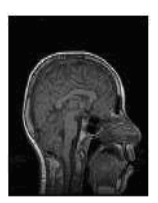

SOURCE

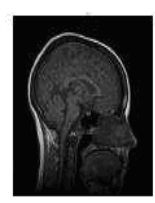

TARGET

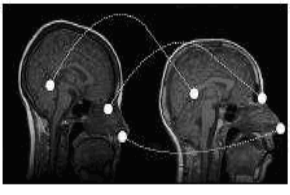

CONTROL POINTS

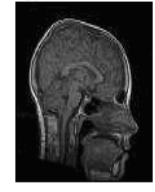

REGISTERED
Figure 1: The Image Registration process.

Steps in Image Registration involve pre-processing, feature selection, feature correspondence, determining and applying a transformation, and in many cases re-sampling of the images [3].

\section{MEDICAL IMAGE REGISTRATION}

Image registration has evolved independently in various research areas, ranging from geo-surveying to medical imaging, each with a number of unique applications. Medical image analysis, in particular, has many challenging and useful applications. The need for registration can arise 
when images of a given piece of anatomy are taken over a period of time and need to be compared, as is the case for the study of tumor growth or in functional magnetic resonance imaging(fMRI) studies [4, 5]. Registration can also be used to characterize normal versus abnormal anatomical shape variations. For example, tumors may be registered to normal or abnormal tumors within the same class. Other applications include labeling and segmentation, in which the image to be labeled/segmented is registered to a previously labeled/segmented image (the atlas).This atlas registration circumvents the need for explicit labeling/segmentation.

The image that needs to be compared may also be obtained using different hardware devices, each highlighting a specific part of the body (these images are also referred to as multi-modality images). Examples of common multimodality images in medical imaging include Computed Tomography (CT), X-ray imaging, Positron Emission Tomography (PET) imaging, and Nuclear Magnetic Resonance (NMR) Imaging. If these images are registered, they can be combined (fused) in a more meaningful way to provide an integrated view.

Medical image registration is a very popular area of image processing with applications ranging from monitoring disease progression [6], building medical atlases [5], image guided surgery etc [4]. A survey of the recent publications in the field of medical image registration with algorithms, validations and applications can be found in [6] and [7]. More details about the feature selection and correspondence, transformation functions and evaluation methods for 2-D and 3-D image registration can be found in [8].

\section{NONRIGID IMAGE REGISTRATION 3.1 Normal or Body Text}

Non-rigid registration is an active area of research in the field of medical image registration. This activity is due to the fact that one cannot decide on the best algorithm for all applications, i.e., no universal solution exists for the mapping problem and lack of a reference standard to compute the exact errors obtained in the registration process [10]. Each algorithm works well under certain constraints and conditions but may not do so under a different set of conditions. Crum et al [9] discusses the various types of non-rigid registration algorithms, their concepts, applications and limitations. The main problem that nonrigid registrations pose is that it is not possible to actually predict the deformation field [9]. The control points may be mapped exactly in the source and target images/volume using different methods [11], but the accuracy in mapping the other points in the source image/volume map onto in the target image/volume is unpredictable. This is because nonrigid registration involves more transformations than just rotation and translation as required for rigid registrations [9]. This work focuses on evaluating a non-rigid registration technique which allows the deformation field to be as-rigidas-possible while still performing local stretching deformations.

\subsection{As-rigid-as-possible Transformations}

The concept of as-rigid-as-possible transformations was first introduced by Alexa et al [12] where an object space morphing technique that blends the interiors of objects as a smooth blending was proposed. This morphing was called as-rigid-as-possible because the objects undergo minimum distortion during morphing. The shape manipulation technique presented by Igarashi et al [13] is also based on as-rigid-as-possible transformations. First Page Copyright Notice

\subsection{MLS Transformations}

The principle of the MLS technique is to minimize the least squares error function obtained during the mapping transformation process. A set of control points are chosen and transformation function is obtained for each point in the image and is based on a weight function included in the least squares error function at each point of evaluation. This weight function ensures that the effect of a control point is seen mostly in the regions immediately surrounding it, while its effect is less prominent in far off regions. The transformation matrix of the MLS technique can include affine, similarity and as-rigid-as-possible transformations and therefore extended here for registration. This work focuses on the as-rigid-as-possible transformations which are capable of producing local deformations. The transformation function is smooth and interpolates the control points.

Given a set of control points on the source and target images, the MLS technique computes the transformation $\mathrm{I}_{\mathrm{v}}(\mathrm{x})$ that best minimizes the least squares error: $\sum\left|I\left(p_{i}\right)-q\right|^{2}$ where $p_{i}$ and $q_{i}$ are the set of control points in the source and target images respectively. This transformation however produces a single affine transformation of the entire image as there is no control over the scaling or shearing in the image. A weighting function included to this least squares error fixes this problem and thus produces a different transformation function for each point of evaluation of the image.

$$
\sum_{i} w_{i} \mid I\left(p_{i}-\left.q_{i}\right|^{2}\right.
$$

The weighting function $w_{i}$ is of the form

$$
w_{i}=\frac{1}{\left|p_{i}-v\right|^{2 \alpha}}
$$

where $v$ is the point of evaluation in the image and $\alpha$ is the fall-of-parameter of the weighting function whose value decides if the weights computed are small or large. The weighting function is dependent on the point of evaluation and thus produces a different transformation for each point of the image. Hence the method is called Moving Least Squares. We can see that as $v$ approaches the control points, the weight approaches infinity and the transformation function interpolates.

The transformation function can be solved as a simple linear transformation matrix, $M$ and a translation vector, $T$ as: 


$$
I_{v}(x)=x M+T
$$

The transformation matrix $M$ can be modified to include affine, similarity and rigid transformations. To perform as-rigid-as-possible transformations, the matrix, $M$ must be constrained to satisfy the condition for rigidity $M^{\prime} M=I$. The translation component can be easily computed by:

$$
T=q_{*}-p_{*} M
$$

where $p_{*}$ and $q_{*} \quad$ are the weighted centroids of the control points given by:

$$
p_{*}=\frac{\sum_{i} w_{i} p_{i}}{\sum_{i} w_{i}} \text { and } \quad q_{*}=\frac{\sum_{i} w_{i} q_{i}}{\sum_{i} w_{i}}
$$

The transformation function can now be calculated as:

$$
I_{v}(x)=\left(x-p_{*}\right) M+q_{*}
$$

The least squares problem can be written as:

$$
\sum_{i} w_{i}\left|\hat{p}_{i} M-\hat{q}_{i}\right|^{2}
$$

where $\quad \hat{p}_{i}=p_{i}-p_{*}$ and $\quad \hat{q}_{i}=q_{i}-q_{*}$

The transformation matrix for the as-rigid-aspossible transformations can be obtained by eliminating the scaling constant. The solution is simple and closed form. It can be obtained easily by a slight modification of the similarity transformation for which the transformation matrix must satisfy the condition, $M_{1}{ }^{\prime} M_{2}=M_{1} M_{2}{ }^{\prime}=\lambda^{2} I$, where $\lambda$ is some constant and $\mathrm{M}_{1}$ and $\mathrm{M}_{2}$ are the columns of $M$ and are vectors of size $2 \times 1 . M_{1}$ and $M_{2}$ have the relationship such that

$$
\text { , } M_{2}=M_{1}^{\perp}
$$

For rigidity condition to be satisfied, $M^{\prime} M=I$, the scaling constants needs to be removed. By using partial derivatives with respect to the free variables in $M$ and substituting the values back into the error function the optimum transformation function is obtained as:

$$
\begin{aligned}
& (x, y)^{\perp}=(-y, x) \\
& M=\frac{1}{\mu_{r}} \sum_{i} w_{i}\left(\begin{array}{l}
\hat{p}_{i} \\
-\hat{p}_{i}{ }^{\perp}
\end{array}\right)\left(\begin{array}{ll}
\hat{q}_{i}^{T} & \left.-\hat{q}_{i}{ }^{\perp T}\right)
\end{array}\right. \\
& \mu_{r}=\sqrt{\left(\sum_{i} w_{i} \hat{q}_{i} \hat{p}_{i}{ }^{T}\right)^{2}+\left(\sum_{i} w_{i} \hat{q}_{i} \hat{p}_{i}{ }^{\perp T}\right)^{2}}
\end{aligned}
$$

removes any scaling and thus produces as-rigid-as possible transforms.

The non-rigid registration technique evaluated in this paper, the MLS transformation is escribed in [11]. Though this paper is build on technique described by Igarashi it aims at achieving faster deformations. For this purpose we propose the transformation of objects by using linear MLS. No triangulation of the input is needed in this case and the transformation can be computed at each point in the image. The transformations achieved using rigidity constraints are as-rigid-as-possible with minimum nonlinear shearing and non-uniform scaling, which is a property of MLS transforms, thus making it a suitable candidate for applications in medical image registration where rigid registrations are insufficient and non-rigid registrations are necessary to align features of the image.

\subsection{Thin Plate Spline Transformations}

Thin-plate splines are a part of the spline family with radial basis functions as the interpolating function. They produce smooth and closed form transformations and have been used extensively in image deformation. Thin-plate Splines have been used in remote sensing for mapping images. Goshtasby et al [8] describes how the thin-plate splines can be used in image deformation for remote sensing images. His book on $2 \mathrm{D}$ and $3 \mathrm{D}$ registrations also describes the TPS transformation function and it applications [14]. Though the TPS produces smooth transformations, the main disadvantage of the technique is that each control point in the image has a global effect on the transformation and thus even if one point is perturbed all other points in the image do not get mapped correctly [9]. The work by Bookstein et al [15] also describes the theory behind TPS transformations and how it can be used in medical image processing. Numerous papers have been published in the medical image processing area using the TPS algorithm in image registration [16, 17]. The TPS algorithm can be used to obtain an elastic transformation to map the source image to the target image. Rohr et al [18] uses the TPS as a technique to obtain elastic registration of brain images taking into account the errors at the landmark points in the images. The method proposed in this paper is applicable to 2D and 3D MR images. This paper establishes that the MLS transformation technique can be used as an alternate to the TPS transformation technique and can perform better due to its as-rigid-as-possible nature. This could enable the MLS technique to be used in applications like constructing medical atlases, studying the progression of diseases, postoperative shift of brain and other such applications where the TPS technique has been used so far.

\section{IMPLEMENTATION DETAILS}

The overall system for the non-rigid registration of the medical images obtained from the same patients using the MLS and TPS techniques is shown in figure 4.1. For each pair of the source and target images first of all a set of homologous control points are extracted. These control points from the source and the target are taken as the input to the Moving least Squares (MLS) and Thin Plate Spline (TPS) algorithms, which will find out a transformation function for each pixel position in the source image to obtain the registered source. In the case of images taken from two different patients, it is difficult to identify homologous control points. As these points play the most important role in the registration process it becomes necessary to accurately obtain homologous control points from the source and target images. Initially two images of the same modality $\mathrm{S}$ and $\mathrm{T}$ from two different patients were given as the input to the system. The two images were registered using an intensity based method. The deformation function thus obtained was applied to the source image $S$ to 
get the new transformed image T. T has homologous points to the source $\mathrm{S}$. The transformed source image $\mathrm{T}$ obtained by the intensity based registration algorithm was used as the new target image for the point based registration process. In this case we can quantitatively evaluate the performance of the two techniques by computing the Target Registration Error(TRE) as shown in the following figure 2.

\section{.4.1. Intensity Based Non-Rigid Registration Algorithm}

Here an error function is estimated using Taylor-series expansion, in order to simplify the minimization. A more accurate estimate of the actual error function can be determined using a Newton-Raphson style iterative scheme. In particular, on each iteration, the estimated transformation is applied to the source image, and a new transformation is estimated between the newly warped source and target image. As few as five iterations greatly improve the final estimate. Secondly, the required spatial/temporal derivatives have finite support, thus fundamentally limiting the amount of motion that can be estimated. A coarse-to-fine scheme is adopted in order to contend with larger motions [22]. A Gaussians pyramid is built for both source and target images and the local affine parameters are estimated at the coarsest level. These parameters are used to warp the source image in the next level of the pyramid. A new estimate is computed at this level, and the process repeated through each level of the pyramid. The transformations at each level of the pyramids are accumulated yielding a single final transformation. This multiscale approach is critical given the differential nature of our measurements, allowing us to register images with larger motions. Finally the estimation of the spatial/temporal derivatives is crucial step. Spatial/temporal derivatives of discretely sampled images are often computed as differences between neighbouring sample values. Such differences are typically poor approximations to derivatives and lead to substantial errors. In computing derivatives we employ a set of derivative filters specifically designed for multi-dimensional differentiation [23]. The spatial/temporal derivatives are estimated as follows. The images are first pre-filtered in time(using the two-tap filter( $\left[\begin{array}{ll}0.5 & 0.5\end{array}\right]$ ). The derivative in $\mathrm{x}$ is then estimated by pre-filtering the result in $\mathrm{y}$ (using the 3-tap pre-filter $\left.\left[\begin{array}{llll}0.223755 & 0.552490 & 0.223755\end{array}\right]\right)$, followed by differentiating in $\mathrm{x}$ (using the derivative filter[$\left.\begin{array}{lll}0.453014 & 0.0 & 0.453014\end{array}\right]$ ). Similarly the derivative in $\mathrm{y}$ is estimated by first pre-filtering the result in $\mathrm{x}$ (using the 3-tap prefilter $\left[\begin{array}{llll}0.223755 & 0.552490 & 0.223755\end{array}\right]$ ), followed by differentiating in y (using the derivative filter [-0.453014 0.0 $0.453014]$ ). The derivative in time is estimated by first prefiltering in space (in $\mathrm{x}$ and $\mathrm{y}$ ) using the 3-tap pre-filter [0.223755 0.5524900 .223755$]$ ), followed by applying the two-tap derivative filter [ 0.50 .5$]$ in time to the result. These filters significantly improve the resulting registration.

System overview for the registration algorithm is split into three sections as depicted in Figure 3. It depicts the multiscale estimation of the registration map, from a coarse to fine scale. The multiscale registration algorithm proceeds as follows. The source and target images at the coarsest scale are registered to obtain an initial estimation of the registration map [10]. This initial estimate is used to warp the source image at the next scale. The warped source image is then registered with its corresponding target image. This process repeated at each level of the pyramid. A single registration map is obtained by accumulating successive estimated registration maps at each scale. This multiscale registration approach allows us to recover large motions as well as small motions. Within each scale, the registration map is determined in an iterative fashion. After an initial estimation of the registration parameters the source image is warped with the estimated parameters and registered again with the target image. During each of these iterations, successive intermediate registration maps are accumulated to form a single registration map [24]. The iterations are stopped when the average displacement of the estimated motion is less than 0.1 pixels. Within each scale, and within each accuracy-iteration, a smooth registration map is obtained as follows. Given source and target image, an estimate of the registration map without smoothness is first obtained.

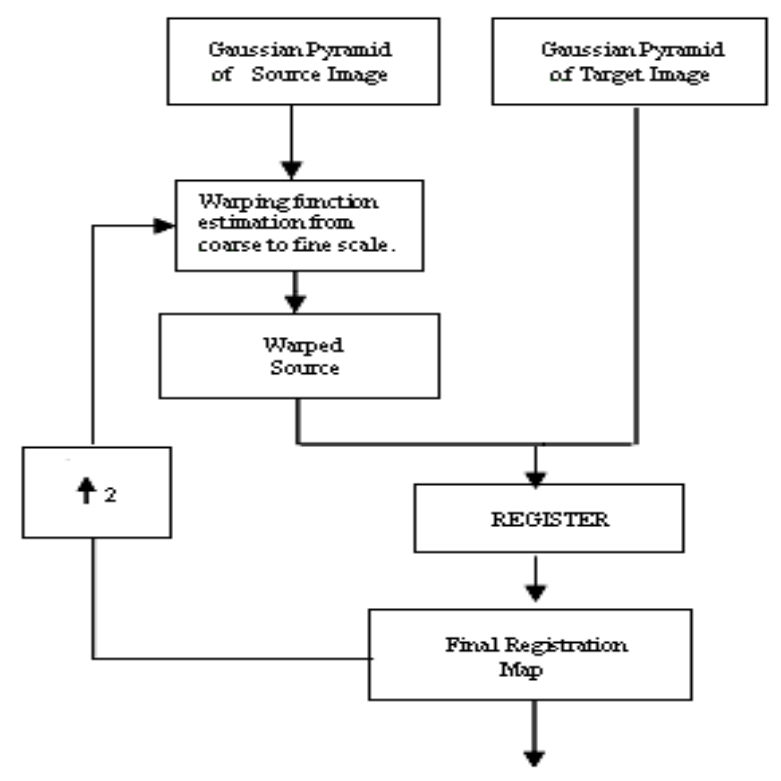

Figure 3: System diagram - Intensity Based Non Rigid Registration

This initial estimate is used to bootstrap the nonlinear iterative estimation of the smooth registration map [22]. These registrations are referred to as smoothnessiterations. These three components together form the complete registration algorithm.

\subsection{Control Point Selection}

Control point selection is an important aspect in point based image registration technique. For good registration results it is necessary to select these control points as accurately as possible. Control points are selected from the source and target images by the user. Anatomically similar structures from the two images are usually selected as the control points. After the selection process it is applied to both the registration algorithm to obtain the registered source. 


\subsection{Target Registration Error (TRE)}

Without quantitative evaluation, no registration method can be accepted for practical utilization. The Target Registration Error (TRE) is one such method which is the displacement between two corresponding points after registration, i.e., after one of the points has been subjected to the registering transformation. A lower TRE indicates a better method. The word target in the name of this error measure is meant to suggest that the error is being measured at an anatomical position that is the target of some intervention or diagnosis. Such errors would be expected to be more meaningful than errors measured at points with no intrinsic clinical significance. Let $\mathrm{p}$ represent a point in the first image of a pair to be registered, and $\mathrm{q}$ a point in the second image. A registration method applied to this pair leads to a transformation $\mathrm{T}$ that, without loss of generality, registers the first image to the second. The difference between the two vectors representing the transformed point and the corresponding point gives the target registration error. Thus, $\mathrm{TRE}=\mathrm{T}(\mathrm{p})-\mathrm{q}$, which is nothing but the registration error at that point and is computed as the disparity in the position of two corresponding points after registration. A low TRE thus indicates a better method.

\section{EXPERIMENTAL RESULTS}

\subsection{Datasets Used}

This section presents the results for evaluation of two dimensional images using the MLS and TPS techniques. We use two dimensional Magnetic Resonance images (MR) of brain from same patients as well as different patients for performing the registration. The datasets used are jpeg images of size $256 \times 256$.

\subsection{Quantitative Analysis- Performance Metrics}

Qualitative (Visual) comparisons are not sufficient to evaluate the performance of any algorithm, quantitative evaluations have to be made. For this purpose the target registration error (TRE) was computed at certain points in the image for both the methods. The target registration error at any point, which is nothing but the registration error at that point, is computed as the disparity in the position of two corresponding points after registration [20,9]. To compute the TRE, three points (namely A, B and C) at random was selected on the internal structures of the brain as shown in the Figure $4(\mathrm{a} \& \mathrm{~b})$. The TRE at these points $\mathrm{A}, \mathrm{B}$ and $\mathrm{C}$, shown on the source image, was computed by finding the points they mapped to on the MLS and TPS registered images. Since the points corresponding to A, B and C could be found on the target image by using the Intensity based non-Rigid registration Algorithm comparisons could be made on the resulting TRE's for the two methods. Table 1 shows the resulting TRE values for the two methods.

\begin{tabular}{|l|c|c|}
\hline Points & TRE $_{\text {ML }}$ & TRE $_{\text {TPS }}$ \\
\hline A (Yellow) & 1.3533 & 1.6567 \\
\hline B ( White) & 1.6432 & 2.2689 \\
\hline C ( Blue) & 1.4065 & 1.7250 \\
\hline
\end{tabular}

Table 1: Comparison of MLS and TPS

Figure 4(a:d) Shows the Registration of 2D MR Brain Images with points marked with Yellow, White and Blue used to calculate TRE.

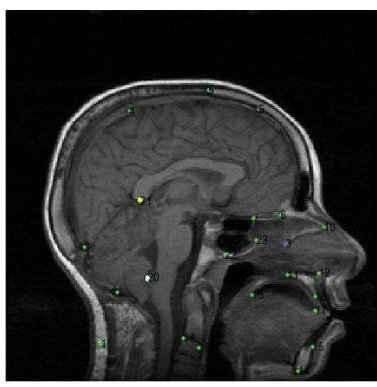

Figure 4 (a)

Source Image

with 21 Control Points

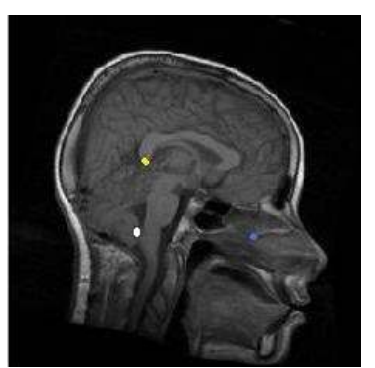

Figure 4 (c)

Registration -MLS

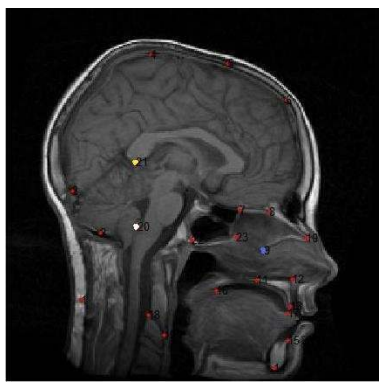

Figure 4 (b)

Target Image with 21 Control Points

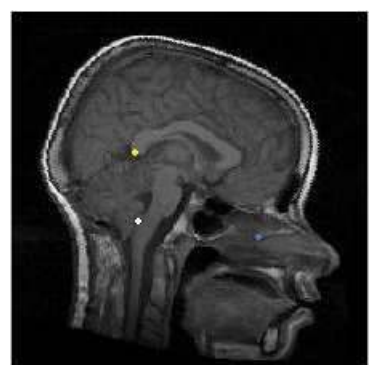

Figure 4 (d)

Registration - TPS

\subsection{Computational Aspects for MLS and TPS}

The MLS and TPS algorithm were implemented in Matlab. The MLS algorithm involves finding the transformation at each point in the image and thus has a longer computation time as seen in Table 2. One way to reduce the computation time would be to decimate the image or volume using a grid and apply the deformation to each vertex of the grid instead of each point and interpolating the other points using bilinear interpolation. TPS transformations are computationally less time consuming.

\subsection{Observations}

If the control points are uniformly distributed in both the source and target, it is difficult to visually analyse the performance of both the algorithms. In this case both the algorithms will give visually similar results. Hence the 
Target Registration Error (TRE) is used as a quantitative metric to evaluate the performance of both the techniques.

\begin{tabular}{|c|l|l|}
\hline $\begin{array}{l}\text { No. of } \\
\text { Pivots } \\
\text { Used }\end{array}$ & $\begin{array}{l}\text { Execution } \\
\text { Time } \\
\text { MLS ( in ms) }\end{array}$ & $\begin{array}{l}\text { Execution } \\
\text { Time } \\
\text { TPS }(\text { in } \mathrm{ms})\end{array}$ \\
\hline 4 & 78 & 8 \\
\hline 9 & 124 & 13 \\
\hline 20 & 235 & 21 \\
\hline 34 & 380 & 30 \\
\hline
\end{tabular}

Table 2: Execution Time for MLS and TPS Algorithm Based on Number of Control Points

A lower TRE indicates a better method. Here three points other than the control points are randomly selected from the source and target. Then the TRE value is calculated. In each case it was found that the TRE for MLS technique is low as compared to TPS. The execution time for both the methods by varying the number of control points indicates that TRE perform faster than MLS. Even if the execution time of TPS is faster compared to MLS, the later will give less error during the registration. So we can conclude that MLS technique is quantitatively better than TPS algorithm.

\subsection{Qualitative Analysis}

Qualitative analysis is done based on Human Perception. To evaluate the performance of the two techniques based on the placement of control points, the homologous control points were selected only on the spine leaving out the other regions as shown in figure 5 (a) and (b). The registration result using MLS and TPS techniques can be seen in figure 5(c) and (d). It was observed that even though control points were not placed in the head region, the MLS technique produced a qualitatively better result. This technique did not show any non-uniform scaling or stretching and also produced an asrigid-as-possible transformation. The TPS transformation was seen to stretch the image unnaturally. In this case too, we observed the stretching caused in the spine region along with the stretching seen near the head region. Thus it can be said that non-uniform placement of control points affects the deformation field minimally in case of the MLS technique than the TPS technique which showed more stretching.
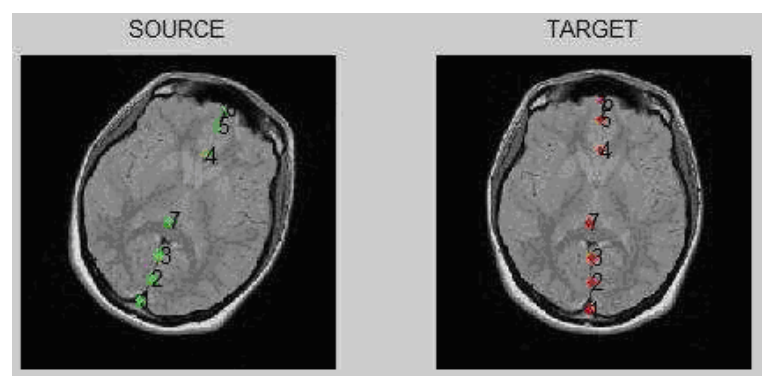

Figure 5 (a) Source and Target Image with 7 Control Points

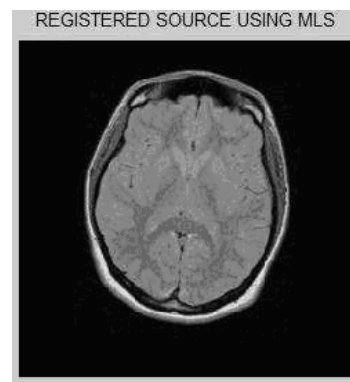

Figure 5 (b)

Registration - MLS

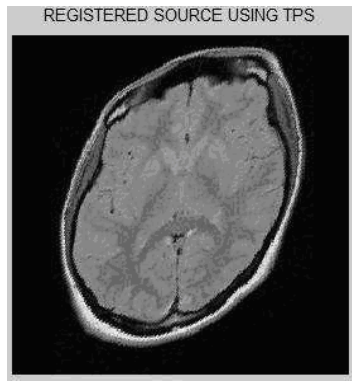

Figure 5 (c)

Registration - TPS
To show that MLS can be used as a better technique for nonrigid registration while maintaining the overall rigidity of the image the two techniques were also evaluated by using a limited number of control points. Using more control points will produce equally good results for the two methods since this allows more control over the deformation field. Figure 6 (a) shows the source and target images with 3 control points placed on the head region. The results of the two registration techniques can be seen in the Figure 6 (b) and (c). A visual inspection of the two transformations showed that the MLS algorithm produces a qualitatively better image than the TPS method.

The MLS transformation was observed to map the source to the target image with minimal stretching, due to its as-rigidas-possible transformation ability, while the TPS transformation was observed to cause more stretching across shape.

If the number of control points selected are less or if they are not uniformly distributed the Thin Plate Spline (TPS) registration algorithm fails. But in both the scenarios MLS technique will produce visually good results than TPS.

\section{CONCLUSIONS}

In this work we have applied an interpolating non-rigid registration technique i.e., Moving Least Squares and compared the results both quantitatively and qualitatively with another point based technique i.e., Thin Plate Splines. MLS technique was found to be a good candidate for nonrigid registration for a given number of control points than thin plate splines. A theoretical nicety of the MLS technique is that the deformation field is as rigid as possible, given the constraint that the control points are to be interpolated.

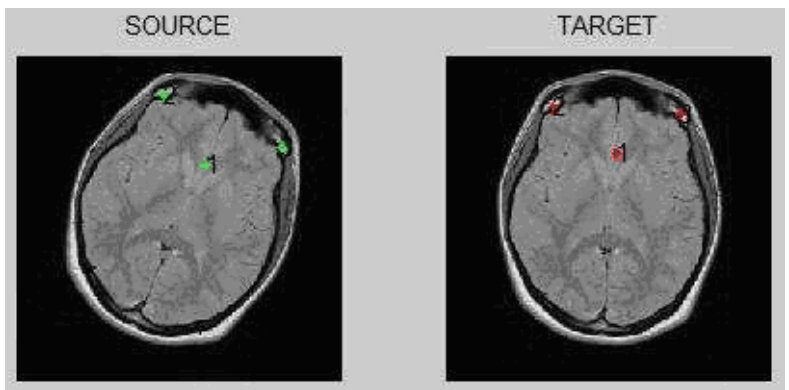

Figure 6 (a) Source and Target Image with 3 Pivots 


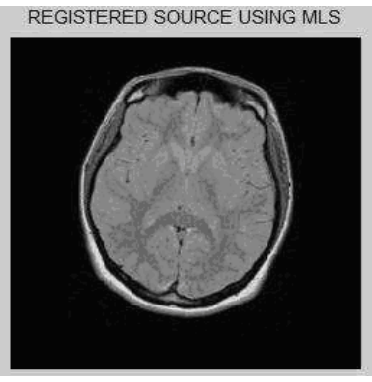

Figure 6 (b)

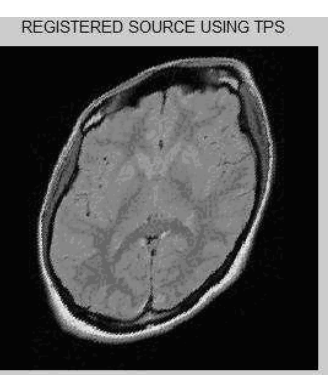

Figure 6 (c)
Registered Source- ML Registered Source -TPS

This can offer advantages over other non-rigid registration methods, particularly those where bony structures should be minimally deformed during registration. Non-rigid registrations often produce unwanted stretching in the images and the unpredictable nature of the deformation field poses a major drawback which makes an algorithm with the ability to produce as-rigid-as-possible transformations attractive. The as-rigid-as-possible nature of the MLS technique thus makes it a suitable candidate for non-rigid registrations as it provides a transformation that maintains the rigidity of structures that need to remain non-deformed, while producing local deformations.

The overall rigidity of the image is maintained better with the MLS method. This can be seen clearly in the example of the 2D MR brain images taken from the same patients. Certain structures of the brain in the source image is deformed to match the target image while the other regions of the image remain minimally affected thus maintaining the global rigidity while performing local deformations. The results show that there are no unwanted stretching and shearing in the image. This is because the effect of the control points on each pixel location is weighted by a weight function. The results for the registration of $2 \mathrm{D}$ MR human brain images of different patients is used for qualitative comparison. A quantitative evaluation is done by comparison of the target registration error (TRE).It shows that the MLS method has lower error values. Selecting the control points forms an important aspect of image registration. Thus it becomes necessary to select these control points as accurately as possible. Any error in this placing of points affects the final registration result. This is more pronounced in case of the TPS transformation as each point has a global effect on the deformation field. From the results obtained for the MLS transformation we observed that the as-rigid-as-possible nature of transformation produces an acceptable deformation even if one or two control points are not placed accurately. This is because of the weighting function on the least squares error function. This weighting function ensures that the effect of the control point in regions far away from it is less affected. The MLS transformation involves computing the transformation at each point in the image or volume. This leads to a longer computation time though it produces good results. One way to reduce the computation time would be to decimate the image with a grid and computing the transformation only at the grid vertices and interpolating the other point in the image using an interpolation technique like bilinear interpolation.

The MLS method can be used for producing even better registration results. In the future we would like to evaluate the performance of the MLS algorithm by modifying the weight parameter. Study can also be made by examining the results by using different distance functions to see the variation of rigidity in the deformation. Another area of interest would be to study the effect of perturbation of the control points. Research can be carried out in future to find out ways for optimizing the MLS algorithm by automating the process of control point selection.

\section{REFERENCES}

[1] J. Maintz and M. Viergever, "A survey of medical image registration," in Medical Image Analysis, pp. 1-36, March 1998.

[2] R. Kolluri, "Provably good moving least squares," in Proc. of International Conference on Information Processing in Medical Imaging, pp. 1008-1017, 2005.

[3] H.Chui and A. Rangarajan, "A new point-matching algorithm for non-rigid registration," in IEEE International Conference on Computer Vision and Image Understanding, pp. $114-141,2003$.

[4] F. Sauer, "Image registration: Enabling technology for image guided surgery and therapy," in IEEE Transactions on Engineering in Medicine and Biology Society, pp. 7242$7245,2005$.

[5] A.W. Toga and P.M. Thompson, Handbook of medical imaging, $1^{\text {st }}$ ed., 2000.

[6] P. Freeborough and N. Fox, "Modeling brain deformations in alzheimer disease by fluid registration of serial 3d mr images," Journal of Computer Assisted Tomogra- phy, vol. 22, pp. 838-844, 1984.

[7] M. Sonka and M. Fitzpatrick, "Handbook of medical imaging," Medical Image Processing and Analysis, SPIE Press Monograph, vol. 22, pp. 838-844, 1984.

[8] A. Goshtasby, "Registration of images with geodesic distortion," in IEEE Transactions on Geoscience and Remote Sensing, pp. 60-64, January 1988.

[9] W. Crum and T. Hartkens, "Non-rigid image registration: Theory and practice," The British Journal of Radiology, vol. 77, pp. 140-152, December 2004.

[10] J. Hajnal and D. Hawkes, Medical Image Registration. CRC Press, 2 ed., 2001.

[11] S. Schaefer and J. Warren, "Image deformation using moving least squares," in ACM Transactions on Graphics, p. 533â540, July 2006.

[12] M. Alexa and D. Levin, "As-rigid-as-possible shape interpolation," in Proceedings of ACM SIGGRAPH, pp. 157-164, 2000.

[13] T. Igarashi and J. Hughes, "As-rigid-as-possible shape manipulation," in ACM Transactions on Graphics, vol. 24, pp. 1134-1141, April 2005. 
[14] A. Goshtasby, "2D and 3D medical registration for medical, remote sensing, and industrial applications," in IEEE Transactions on Geoscience and Remote Sensing, vol. 26, pp. 80-84, April 2005.

[15] F. Bookstein, "Principal warps: Thin-plate splines and the decomposition of deformations," in IEEE Transactions on Pattern Analysis and Machine Intelligence, pp. 567-585, June 1989.

[16] H. Park and C. Meyer, "Construction of an abdominal probabilistic atlas and its application in segmentation," in IEEE Transactions on Medical Imaging, pp. 483-492, April 2003.

[17] H.J.Johnson and G. E. Christensen, "Landmark and intensity based, consistent thin-plate spline image registration," in Proc. of International Conference on Information Processing in Medical Imaging, vol. 2082, pp. 329-343, 2001.

[18] K. Rohr and M. H. Kuhn, "Landmark-based elastic registration using approximat- ing thin-plate splines," in IEEE Transactions on Medical Imaging, pp. 483-492,2001.

[19] Y. Zhu and S. Gortler, "3d deformation using moving least squares," Harvard Computer Science Technical Report, pp. 10-17, 2007.
[20] K. Rohr and M.Fornefett, "Spline-based elastic image registration: integration of landmark errors and orientation attributes," in IEEE Transactions on Computer Vision and Image Understanding, pp. 483-492, 2003.

[21] G. Rohde and B. Dawant, "The adaptive bases algorithm for intensity-based non- rigid image registration," in IEEE Transactions on Medical Imaging, pp. 483-492, April 2003.

[22] Senthil Periaswamy, Hany Farid:"Medical Image Registration with Partial Data" ,IEEE Transactions on Medical Imaging 2006.

[23] C.Davatzikos, "Spatial transformation and registration of brain images using elastically deformable models," Computer Vision and Image Understanding: CVIU, 66(2):207-222, 1997.

[24] L. Zagorchev and A. Goshtasby, "A comparitive study of transformation functions for nonrigid image registration," IEEE Transactions on Image Processing, 15(3):529 - 538, March 2006.

[25] S.Periaswamy and H.Farid, "Elastic registration in the presence of intensity variations," IEEE Transactions on Medical Imaging," 22(7):865-874, July 2003. 